\title{
A Robust Compositional Architecture for Autonomous Systems
}

\author{
Guillaume Brat, Ewen Denney, Kimberley Farrell, Dimitra Giannakopoulou, Ari Jónsson \\ Research Institute for Advanced Computer Science \\ NASA Ames Research Center, Mailstop 269-2 \\ Moffett Field, CA 94035 \\ \{brat, edenney, kfarrell, dimitra, jonsson\}@email.arc.nasa.gov \\ Jeremy Frank \\ NASA Ames Research Center, Mailstop 269-2 \\ Moffett Field, CA 94035 \\ frank@email.arc.nasa.gov \\ Mark Boddy, Todd Carpenter \\ Adventium Enterprises \\ 100 Mill Place \\ 111 Third Avenue South \\ Minneapolis, MN 55401 USA \\ \{mark.boddy, todd.carpenter\}@adventiumenterprises.com \\ Tara Estlin, Mihail Pivtoraiko \\ Jet Propulsion Laboratory M/S 126-347 \\ 4800 Oak Grove Drive \\ Pasadena CA 9110, USA, \\ \{tara.estlin, mihail.n.pivtoraiko\}@jpl.nasa.gov
}

\begin{abstract}
Space exploration applications can benefit greatly from autonomous systems. Great distances, limited communications and high costs make direct operations impossible while mandating operations reliability and efficiency beyond what traditional commanding can provide. Autonomous systems can improve reliability and enhance spacecraft capability significantly. However, there is reluctance to utilizing autonomous systems. In part, this is due to general hesitation about new technologies, but a more tangible concern is the reliability and predictability of autonomous software.
\end{abstract}

In this paper, we describe ongoing work aimed at increasing robustness and predictability of autonomous software, with the ultimate goal of building trust in such systems. The work combines state-of-the-art technologies and capabilities in autonomous systems with advanced validation and synthesis techniques. The focus of this paper is on the autonomous system architecture that has been defined, and on how it enables the application of validation techniques for resulting autonomous systems.

\footnotetext{
${ }_{1} 0-7803-9546-8 / 06 / \$ 20.00 \bigodot 2006$ IEEE

2 IEEEAC paper \#1499, Version 9, Updated Dec 16, 2005
}

TABle OF CONTENTS

1. INTRODUCTION ...................................................... 1

2. AN ARCHITECTURE FOR AUTONOMY ................. 2

3. VALIDATION OF AUTONOMOUS SYSTEMS............. 4

4. CONCLUDING REMARKS ......................................... 5

REFERENCES........................................................ 6

BIOGRAPHY ........................................................ 7

\section{INTRODUCTION}

Space exploration applications offer a unique opportunity for the development and deployment of autonomous systems, due to limited communications, great distances, and high cost of direct operation. At the same time, the risk and cost of space missions leads to reluctance to taking on new, complex and difficult-to-understand technology. Consequently, there is a pressing need to address the issue of designing robust architecture for autonomous systems and demonstrate a design process that can provide the trust and reliability that is required for manned and unmanned space applications.

In this paper, we describe an ongoing effort to develop a new approach to defining, implementing and maintaining compositional autonomous systems. There are two key elements to the approach. One is a modular compositional autonomy architecture where adaptation to different 
applications is done in an incremental manner. The other is a testing and validation methodology that allows the certification of new adaptations to be limited to the components and relations that are modified. Together, the two elements will make future autonomy applications more easily constructed and modified, while increasing reliability and reducing cost of reconfiguration and maintenance.

The work will be grounded in a specific autonomy architecture that integrates the EUROPA planning framework and the functional layer of the CLARAty control architecture. EUROPA supports incremental compositional specification of the states, commands and associated operations rules that define how it may control a given system. CLARAty provides a compositional approach to defining the functional control software that interfaces with the underlying system.

Compositional verification techniques will be used to limit the efforts required to validate and certify a new adaptation. These methods use known properties of unchanged modules to limit validation and certification efforts to changes made. The validation of core system and individual component properties is done with both formal and empirical analysis.

Our approach will enable the increased use of autonomy in future space explorations, thus reducing operations costs and increasing reliability. In addition, the methodology of composable components and associated incremental testing and verification, will reduce the cost of system development, maintenance, and reconfiguration.

\section{AN ARCHITECTURE FOR AUTONOMY}

Autonomous systems vary greatly in the representation and reasoning techniques utilized in such systems. Furthermore, the interface between autonomous control and underlying systems can be radically different between architectures. Both of these aspects impact the application of validation techniques to autonomous systems instantiations. Consequently, we define and use a general autonomous systems architecture that uses specific representation and reasoning approaches, combined with a structured welldefined interface to the underlying system. While the architecture provides a basis for defining validation processes and techniques, many of the general notions of how to validate autonomous systems will be applicable to other architectures.

Our architecture uses a constraint-based planning framework called EUROPA (Extendible Uniform Remote Operations Planning Architecture) for the core representation and reasoning. This provides the ability to make decisions about what actions to take so as to achieve mission goals, while ensuring that flight rules and constraints are satisfied. The actions are implemented in the CLARAty framework (Coupled-Layer Architecture for Rover Autonomy), which also provides structured access to system states and sensory information. The architecture is shown in Figure 1.

The decision-making component, implemented in EUROPA, uses the domain model to generate safe plans and decisions that respect flight rules and other constraints on operations. The domain model is a declarative specification of actions and states implemented in the functional layer, along with rules on how these actions can

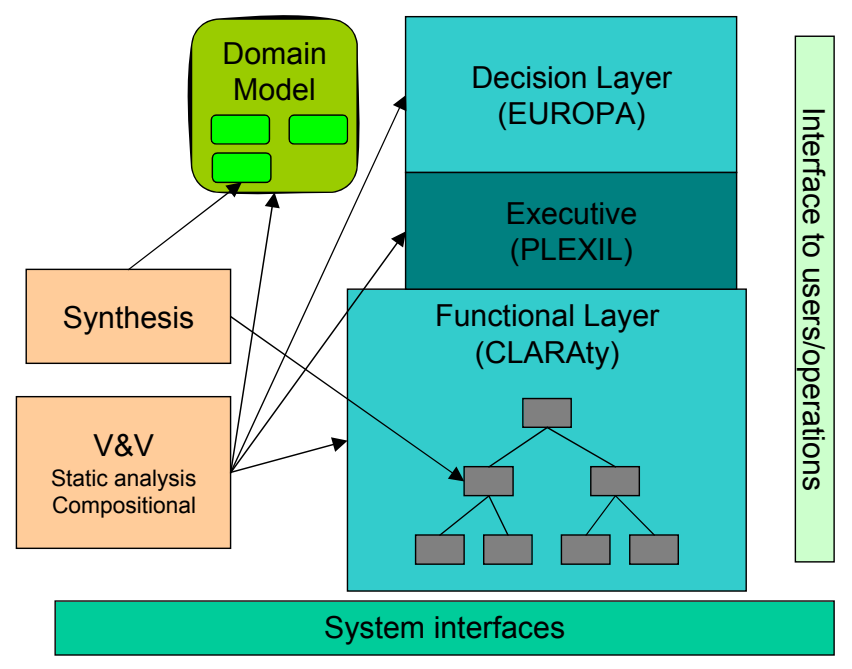

Figure1: Autonomy architecture outline

be used. The domain model is compositional, meaning that new actions and rules can be added without changing existing content. The executive, implemented in an execution framework called PLEXIL, executes the plans and actions specified by the decision layer. It also monitors the execution, ensuring that the constraints and assumptions in the given plan are satisfied in the system during execution. When deviations occur, the executive can either recover or call on the decision layer to decide how to proceed. The functional layer is part of the CLARAty framework. It is a set of functional components, arranged in a hierarchy where higher-level components utilize capabilities and services of lower-level components. The functional layer thus provides a compositional approach to implementing interfaces to system functions.

The verification and validation methods are applied to all levels of the architecture. The details are further described here below in the section on validation. The automated synthesis techniques are then used to generate, from highlevel specifications, both CLARAty functional layer 
components and the associated specifications in the domain model. This approach, combined with the compositional nature of the overall architecture, enables rapid adaptation and reconfiguration of the autonomy system.

\section{EUROPA - constraint-based planning}

The Extensible Universal Remote Operations Planning Architecture (EUROPA) is a model-based planning and scheduling architecture descended from the Remote Agent Planner [14] . Users of EUROPA can specify the rules of planning domains using a rich domain description language that supports time, resources, disjunctive preconditions and conditional effects. EUROPA makes extensive use of constraint based representation and reasoning, which allows for more concise representation of planning models, and more efficient reasoning during planning [11] . EUROPA provides support for "foreign function" calls implementing complex constraints such as power consumption and generation.

EUROPA consists of a hierarchy of highly configurable components, supporting the building many types of planners and plan representations. The modeling language, NDDL, contains a small number of elementary entity types, providing ease in modeling. These types can be extended to provide more specialized components, leading to a rich set of modeling primitives. The plan database contains the current plan and information about its state. The database provides mechanisms to efficiently query the plan state and modify the plan. Modification leads to inference, which is performed by a rules engine module and a constraint reasoning engine module. The rules engine determines which rules in the domain description apply after each modification of the plan, and updates the state accordingly. The constraint reasoning module is further broken down into specialized modules that efficiently handle particular classes of constraints, such as temporal constraints. Finally, EUROPA provides interfaces to specialized heuristics modules that provide search control to planners.

EUROPA can also be customized to support both longrange deliberative planners as well as short-horizon continuous planners that may operate on the same model. This approach partially resolves problems due to building multiple models in different languages for the same autonomy system (e.g. [12] ). For example, one planner may have a time horizon limited to 5 minutes into the future, and can delay subgoals. Another planner may only plan activities for a hazard avoidance system, leaving other goals to other planners. EUROPA supports customizations of this form by limiting a planners' "view" to a subset of the model. EUROPA also allows multiple planners to modify the same plan concurrently, by providing authority mechanisms indicating what planners may modify.
Automated planning technology such as EUROPA has been utilized as part of on-board autonomy architectures for deep space probes [14], robotic rovers [16] and free-flying robots [17].

\section{CLARAty - layered architecture for robotics}

Most robotic control systems employ a variant of the ThreeLayer Architecture pioneered by Brooks in 1987. CLARAty is an evolution of the three-layer architecture that provides a wide-range of robotic functionality and simplifies the integration of new technologies on robotic platforms. CLARAty is a joint project between the NASA Jet Propulsion Laboratory, NASA Ames Research Center, Carnegie Mellon University and a number of other universities and has been designed specifically for spacebased robotic control applications. CLARAty features a Functional Layer of robotic primitives, coupled with a Decision Layer of planning and execution functionality; each of these layers contains a hierarchy of components ranging from the most elementary to the most "intelligent".

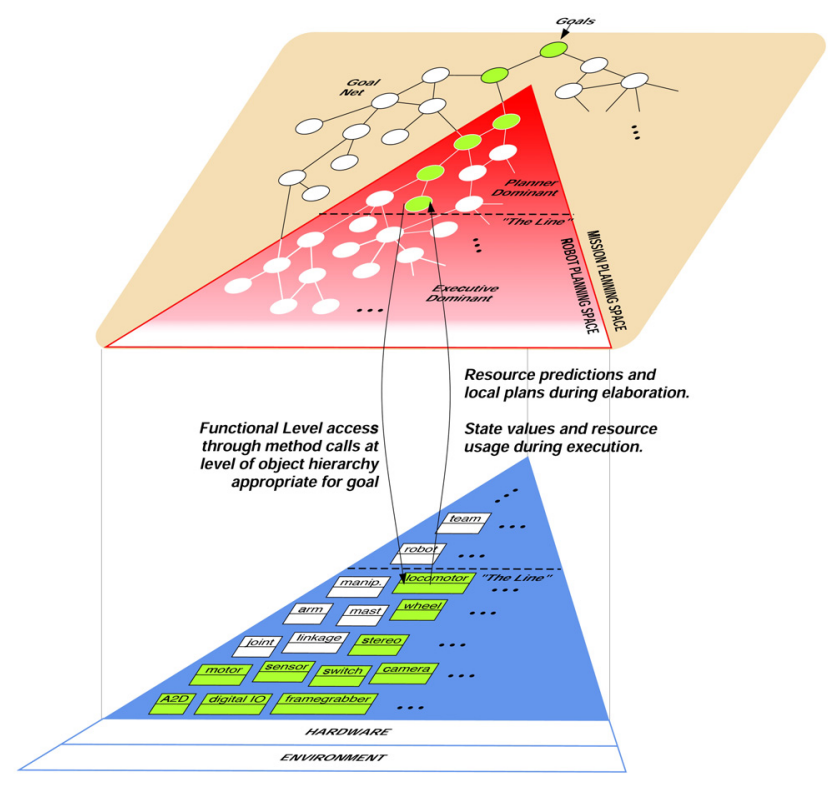

Figure 2: CLARAty framework organization

The Functional Layer (FL) provides a set of standard, generic robot capabilities that interface to system hardware. These capabilities are organized as a software class hierarchy of robotic components; for example, wheeledmobility is a subclass of mobility, and individual rover wheel assemblies are child classes. As is natural in objectoriented systems, the interface is separated from implementation. Physical limitations of devices are distinguished from algorithmic limitations. Finally, runtime models of devices are incorporated in the Functional Layer. 


\section{VALIDATION OF AUtonomous Systems}

In the autonomy architecture outlined here, an instantiation of an autonomous system consists of the following elements:

(1) The core EUROPA planning and decision-making framework, which will largely be unchanged between applications and thus can be validated without reference to the specific instantiation.

(2) The CLARAty instantiation for the system in question, which consists of a set of core CLARAty components and the specific components used to operate the system. The core CLARAty components can be validated separately, while the specific components are validated as part of the instantiation process. Some components may be synthesized, which offers an opportunity for easier validation of those components.

(3) The execution system that links CLARAty and EUROPA and provides monitoring capabilities to ensure that execution does not continue when the assumptions supporting the plan no longer hold. The core execution system is validated once, but online validation and checking techniques can be used to validate specific executable plans.

(4) The domain model describing possible actions and the flight rules governing those actions and the related system states. Model validation is a key element of ensuring that the autonomy system instantiation is robust and safe.

(5) The properties that should hold for the system and various components. These define the criteria for validation of the system.

The validation of an instantiation thus involves validating core architecture systems, instantiation-specific CLARAty components, the domain model used by the planner and executive, and finally, the overall properties for the instantiated system. To tackle this, we apply three kinds of techniques. Model-based validation, using compositional verification, can be applied to core software as well as special-purpose components. In addition, compositional techniques allow us to verify system-level properties from component properties. Static analysis is a powerful technique to directly analyze software code, without requiring a formal modeling of the software components and properties. Finally, automated synthesis techniques allow us to generate instance-specific elements from highlevel specifications. In addition to simplifying the process of implementing instantiations, synthesis offers an additional level of validation by generating provably safe code.

\section{Compositional verification}

Model-based verification techniques use exhaustive search through possible execution trajectories to verify desired system properties. While these techniques can provide the formal validation desired for our autonomous systems, they suffer from state-space explosion. As a consequence, they are typically used to verify relatively small components of an entire system, rather than the system itself. In addition, they do not lend themselves to incremental validation, as we desire to do for instantiations of autonomous systems.

To address these issues, we turn to compositional verification techniques. Compositional verification decomposes the properties of a system into properties of its components, so that if each component satisfies its respective property, then so does the entire system. Components are thus model checked separately. Assumeguarantee reasoning is a promising compositional verification approach, the basic idea behind it being as follows: Consider a system consisting of two components $\mathrm{X}$ and $\mathrm{Y}$. The desire is to prove that a property $\mathrm{P}$ is satisfied by the overall system $\mathrm{X} \mid \mathrm{Y}$. In compositional verification, this is done by identifying an intermediate property A, called an assumption, and using that to split the validation into two smaller problems. The first part is to prove that given $\mathrm{A}, \mathrm{X}$ satisfies $\mathrm{P}$, and the second part is to prove that $\mathrm{Y}$ satisfies (or guarantees) $\mathrm{P}$.

This notion can be utilized in different ways. If the $\mathrm{X}$ and $\mathrm{Y}$ components are already validated, it is likely that the assumption $\mathrm{A}$ is already known and the compositional verification techniques can be applied directly. This is likely to be the case in situations such as where new CLARAty components are being added on top of existing ones. The properties of the core components are known and validated, so the new components can be validated against these proven assumptions.

A more interesting case is when the assumption $\mathrm{A}$ is not known. To address that, we turn to techniques for automatically generating such assumptions from the components $\mathrm{X}$ and $\mathrm{Y}$ and the desired property $\mathrm{P}$. Recently developed techniques make this possible, and allow both the automatic construction of a weakest valid assumption for a given component [19], and of an assumption generated in an iterative fashion through the use of an automata learning algorithm [20] . The assumptions generated by [20] do not need to be the weakest. In fact, the iterative framework converges to the weakest assumption, but may terminate early, if it finds an assumption that is sufficient to prove that $\mathrm{X} \mid \mathrm{Y}$ satisfies $\mathrm{P}$. The framework also guarantees that the 
assumptions that it generates have at most as many states as the weakest assumption for X.

As outlined above, we rely heavily on these compositional verification techniques in our work, both to address computational cost issues, and to enable incremental validation of autonomy system instantiations.

\section{Static analysis}

The goal of static program analysis is to assess properties of a program without executing the program. Several techniques can be used to perform static analysis. Theorem proving, data flow analysis, constraint solving, and abstract interpretation are among the most popular. Generally speaking, a static program analyzer infers properties about the execution of the program from its text (the source code) and a formal specification of the semantics of the language (which is typically built in the analyzer). Static program analyzers are in general excellent for detecting runtime errors.

Runtime errors are errors that cause exceptions at runtime. Typically, in $\mathrm{C}$, either they result in creating a core dump or they cause data corruption that may cause crashes. The main classes of runtime errors are accesses to un-initialized variables, accesses to un-initialized pointers, out-of-bound array accesses, arithmetic underflow/overflow, invalid arithmetic operations, non-terminating loops, and nonterminating calls.

In general, static program analyzers aim at checking all execution paths, sometimes at the cost of incompleteness (i.e., impossibility of determining the safety of all operations with exact precision). In other words, the analyzer can raise false alarms on some operations that are actually safe. However, if the analyzer deems an operation safe, then errors cannot occur on any execution path. The program analyzer can also detect certain runtime errors which occur every time the execution reaches some point in the program.

Traditionally, there are two complementary uses of a program analyzer:

(1) as a debugger that detects runtime errors statically without executing the program, and

(2) as a preprocessor that reduces the number of potentially dangerous operations that have to be checked by a traditional validation process (code reviewing, test writing, and so on).

The first use is akin to traditional debugging; the developer tries to flush as many as bugs as he can from the code before it gets to verification. The second use is called certification; the goal is to prove the absence of errors of a certain class, thus, alleviating the need for testing for this class of errors. This requires that the static analyzer achieves a good selectivity - the percentage of operations which are proven to be safe by the program analyzer. Indeed, if $50 \%$ of all operations in the program are marked as potentially dangerous by the analyzer, there are no benefits to using such techniques.

\section{Automated synthesis}

The overall aim of our work is to be able to reliably reconfigure components in the functional layer of the autonomy architecture. The previous sections have described $\mathrm{V} \& \mathrm{~V}$ techniques that are able to verify that components are free of bugs. Another approach is to generate the components in an inherently trustworthy manner. We are developing the use of automated code generation (also known as program synthesis) for this Control software is particularly appropriate for code generation since it can be modeled concisely at a high-level, while the code which implements it tends to be idiomatic.

A code generator takes as input a domain-specific highlevel description of a task (e.g., a set of differential equations) and produces optimized and documented lowlevel code (e.g., $\mathrm{C}$ or $\mathrm{C}++$ ) that is based on algorithms appropriate for the task (e.g., the extended Kalman filter). This automation increases developer productivity and, in principle, prevents the introduction of coding errors.

AutoFilter [18] is a domain-specific program synthesis system that generates customized Kalman filters for state estimation tasks specified in a high-level notation. AutoFilter's specification language uses differential equations for the process and measurement models and statistical distributions to describe the noise characteristics. It can generate code with a range of algorithmic characteristics and for several target platforms. The tool has been designed with reliability of the generated code in mind and is able to automatically certify that the code it generates is free from various error classes (most are programming error, while some address functional concerns) using automated theorem proving. Since documentation is an important part of software assurance, AutoFilter can also automatically generate various human-readable documents, containing both design and safety related information

\section{CONCLUDING REMARKS}

The work described in this paper is an ongoing effort. The architecture has been defined and an initial version has been implemented. The process for validating new instantiations has been defined and initial efforts are underway to apply 
static analysis, compositional verification and automated synthesis to parts and aspects of the autonomy architecture.

The autonomy software has been adapted to operating JPL's FIDO rover in simulation. As expected, the integration of EUROPA and CLARAty has been straightforward, assisted by the use of the PLEXIL execution framework. At this point, the planner domain model is constructed manually from the specification of the CLARAty interface for FIDO control. Future efforts in the use of synthesis will focus on automatically generating domain model information from functional layer specifications, but the expectation is that engineering experts will still refine the domain model to specify the desired constraints and flight rules.

Static analysis is being applied to modules of the EUROPA framework, providing initial analysis for the current implementation. In the near future, this work will be extended to other modules of EUROPA and to components of the CLARAty framework.

Compositional verification techniques are being tested on models of autonomous rendezvous and docking systems. The results of this work are very promising, having demonstrated the ability to generate small assumptions that in turn enable very fast validation of system properties. These results are in stark contrast to the large amount of time and computing resources needed to apply traditional model checking methods to the full model without the benefit of decomposition.

Automated synthesis techniques for generating Kalman filters from formal specifications have been adapted to generate Kalman filter components for CLARAty. Since such filters play a key role in interpreting sensor information to determine state information like rover location, this is a significant step forward.

To summarize, the effort outlined here has only recently been started, but even at this early point in time, the results are promising. The goal of this work is to provide a robust verified core autonomy architecture, along with the process and tools needed to adapt it to spacecraft operations applications, such that the instantiated autonomous control system is validated and robust.

\section{REFERENCES}

[1] I.A. Nesnas, A. Wright, M. Bajracharya, R. Simmons, T. Estlin, Won Soo Kim, "CLARAty: An Architecture for Reusable Robotic Software," SPIE Aerosense Conference, April 2003.

[2] B. Fischer, J. Schumann, "AutoBayes: A System for Generating Data Analysis Programs from Statistical Models. Journal of Functional Programming, Vol. 13, No. 3, May 2003, pp. 483-508.

[3] J. Whittle, J. Schumann, "Automating the Implementation of Kalman Filter Algorithms," Accepted for publication in ACM Transactions on Mathematical Software (TOMS).

[4] P. Gluck, G. Holzmann, "Using Spin Model Checking for Flight Software Verification," Procedding of 2002 Aerospace Conference, March 2002.

[5] D. Giannakopoulou, C. Paraseanu, H. Barringer, "Component Verification with Automatically Generated Assumption," Journal of Automated Software Engineering, Vol. 11, Kluwer, 2004.

[6] B. Blanchet et al. "Design and implementation of a special-purpose static program analyzer for safety-critical real-time embedded software." LNCS 2566, pp. 85-108, 2003.

[7] Venet "Non-uniform Alias Analysis of Recursive Data Structures and Arrays." In SAS'02, LNCS 2477, pp. 36-51, 2002.

[8] Venet, G. Brat, "Precise and Efficient Static Array Bound Checking for Large Embedded C Programs," Proceedings of PLDI 2004, Washington, D.C., June 2004.

[9] Venet, "A Scalable Nonuniform Pointer Analysis for Embedded Programs," Proceedings of the International Static Analysis Symposium, SAS 04, Verona, Italy. LNCS 3148, Pp. 149-164, Springer 2004.

[10] J. R. Buch, E.M. Clarke, K.L.McMillan, D.L. Dill, J. Hwang, "Symbolic Model Checking: 10E20 states and beyond," In LICS, 1990.

[11] J. Frank and A. Jonsson, "Constraint-Based Attribute and Interval Planning." In the Journal of Constraints, vol. 8, no. 4, 2003.

[12] N. Muscettola and P. Nayak and B. Pell and B. Williams, "Remote Agent: To Boldly Go Where No AI System Has Gone Before." Artificial Intelligence,103(1-2), 1998.

[13] J. Frank, "Bounding the Resource Availability of Partially Ordered Events with Constant Resource Impact", In Proceedings of the 10th International Conference on Principles and Practices of Constraint Programming, 2004.

[14] A. Jonsson and P. Morris and N. Muscettola and K. Rajan and B. Smith, "Planning in Interplanetary Space: Theory and Practice." Outstanding Application Award winner. 
Proceedings of the International Conference on Artificial Intelligence Planning and Scheduling, 2000.

[15] V. Verma and T. Estlin and A. Jonsson and C. Pasareanu and R. Simmons. Plan Execution and Interchange Language (PLEXIL) for Executable Plans and Command Sequences. Proceedings of the $9^{\text {th }}$ International Symposium on Artificial Intelligence, Robotics and Automation in Space, 2005

[16] M. Bernardine Dias and S. Lemai and N. Muscettola. A Real-Time Rover Executive Based on Model-Based Reactive Planning. Proceedings of the $7^{\text {th }}$ International Symposium on Artificial Intelligence, Robotics and Automation in Space, 2003

[17] N. Muscettola and G. Dorais and C. Fry and R. Levinson and C. Plaunt. IDEA: Planning at the Core of Autonomous Reactive Agents. Proceedings of the $3 \mathrm{~d}$ International NASA Workshop on Planning and Scheduling for Space, 2002

[18] E. Denney, B. Fischer, J. Schumann, and J. Richardson. "Automatic certification of Kalman filters for reliable code generation." In Proceedings of the IEEE Aerospace Conference, Big Sky, Montana, 2005. IEEE.

[19] D. Giannakopoulou, C. S. Pasareanu, H. Barringer, Component Verification with Automatically Generated Assumptions, J. of Automated Software Engineering, 2005

[20] J. M. Cobleigh, D. Giannakopoulou, C. S. Pasareanu, Learning Assumptions for Compositional Verification, in Proc. 9th International Conf. on Tools and Algorithms for the Construction and Analysis of Systems, 2003.

\section{BIOGRAPHIES}

Dr. Guillaume Brat received his M.Sc. and Ph.D. in Electrical \& Computer Engineering in 1998 (The University of Texas at Austin, USA). His thesis defined a (max,+) algebra to model and evaluate non-stationary, periodic timed discrete event systems. Since then, he has specialized on the application of static analysis to software verification. From 1997 to June 1999, he worked at MCC where he led a project that developed static analysis tools for software verification. In June 1999, he joined the Automated Software Engineering group at the NASA Ames Research Center and focused on the application of static analysis to the verification of large software systems. For example, he co-developed and applied static analysis tools based on abstract interpretation to the verification of software for the Mars PathFinder, Deep Space One, and Mars Exploration Rover missions at JPL, various International Space Station controllers at MSFC, and the International Space Station Biological Research Project at the NASA Ames Research Center.

Dr. Ewen Denney received his PhD from the Laboratory for the Foundations of Computer Science in Edinburgh, Scotland in 1999 and has subsequently worked in formal methods groups in France and Hong Kong. He has published over 30 papers in the areas of automated code generation, software modeling, software certification, and the foundations of computer science. He has been with the Robust Software Engineering group at NASA Ames since 2002, working on the AutoBayes and AutoFilter program synthesis projects. He is a contractor with the Research Institute for Advanced Computer Science (RIACS)..

Dr. Dimitra Giannakopoulou has been a RIACS research scientist at the NASA Ames Research Center since August 2000. Her research focuses on scalable specification and verification techniques for NASA systems. In particular, she is interested in incremental and compositional model checking based on software components and architectures. She holds a Ph.D. in Distributed Computing from Imperial College, University of London. She has authored over 25 peer-reviewed technical publications. She acts as a regular reviewer for scientific journals and has been a program committee member for several international conferences.

Kim Farrell is a software project manager with over ten years of experience leading scientific and commercial $R \& D$ teams. She has contributed to successful NASA projects in the areas of autonomous systems, spoken dialogue systems, and computational fluid dynamics. Her interests include strategies for developing inherently testable systems and methods to reduce the cost of software maintenance.

Dr. Ari Jónsson received his Ph.D. in Computer Science from Stanford University in 1997. He is currently a senior research scientist with the Research Institute for Advanced Computer Science, and a member of the Planning and Scheduling Group at NASA Ames Research Center. His research and development efforts include work on constraint reasoning, planning and scheduling, robust plan execution, mixed-initiative planning, autonomous operations, and validation of autonomous systems. Currently, Dr. Jónsson is the principal investigator on two technology research and development projects. He has received a number of recognitions and awards for his work at Ames Research Center, including ones that recognize his contributions to the Remote Agent experiment, which took 
place on board Deep Space One in May 1999, as well as recognitions for his work on the infusion of automated reasoning and planning technology into the Mars Rover Exploration mission in 2004.

Dr. Jeremy Frank received his Ph.D. in Computer Science from the University of California, Davis in 1997. He leads the Planning and Scheduling Group at NASA Ames Research Center. He has worked at NASA Ames Research Center since 1997. He is a former member of the EUROPA team, and has worked on NASA scheduling projects for satellite constellations, airborne telescopes, and Deep Space Network communications. He has served as a reviewer for Artificial Intelligence Journal, the Journal of Artificial Intelligence Research, and the Journal of Scheduling, and has served on the program committee for numerous international conferences and workshops. His research interests are automated planning, constraint satisfaction and optimization.

Dr. Mark Boddy is an internationally recognized expert in several areas of computer science research, including planning and scheduling, automated reasoning, and constraint satisfaction. During the past seventeen years he has published more that 30 peer-reviewed articles in journals and conference proceedings, presented tutorials on temporal reasoning and constraint-based reasoning at international conferences, and given numerous invited talks. Dr. Boddy continues to play an active role in the research community, serving on program committees and reviewing for national and international conferences, as well as serving as a journal reviewer for Artificial Intelligence Journal, Computational Intelligence, IEEE Transactions on Pattern Recognition and Machine Intelligence, and the Journal of Artificial Intelligence Research. He has helped to organize numerous workshops and conferences in related research areas, and served as a program reviewer for NASA, the National Science Foundation, and the Air Force Office of Scientific Research.

Mr. Todd Carpenter has over 19 years of systems development and engineering experience for high-value, real-time, fault-tolerant, and secure systems in domains including medical, military and commercial avionics, space, and petrochemicals. His focus has covered detailed hardware and software design, architecture development, systems design and specification, and tools, standards, and processes for enhancing the design flow. He is a certified Six Sigma Green Belt in both design and growth processes. He formerly served as a research and development advisor at Guidant Corporation, where he provided systems engineering for new product developments in emerging, FDA-regulated, markets. As a Honeywell research scientist and business development lead until 2003, Mr.| Carpenter led a Critical Infrastructure Protection thrust, including developing business plans, market studies, and threat analysis based on military, insurance, and industry surveys, which ultimately led to development of a comprehensive macroscopic vulnerability analysis process to identify, evaluate, and rank broad area system vulnerabilities.

Dr. Tara Estlin is a senior member of the Artificial Intelligence Group at the Jet Propulsion Laboratory where she performs research and development of planning and execution systems for rover automation and multi-rover coordination. Dr. Estlin currently leads several efforts that area developing capabilities for onboard rover-command generation, resource planning and scheduling, execution, and data analysis for single and multiple rovers. She is also currently a rover planner for the Mars Exploration Rover mission. Dr. Estlin received a B.S. in computer science in 1992 from Tulane University, an M.S. in computer science in 1994 and a Ph.D. in computer science in 1997, both from the University of Texas at Austin

Mihail Pivtoraiko is with the Advanced Robotic Controls group at JPL. He is one of the engineers of the multiinstitutional robotic software architecture (CLARAty), as well as a collaborator on several robotics research tasks. Mihail completed his M.S. degree at the Robotics Institute, Carnegie Mellon University in 2005, where he participated in several large mobile robotics projects, in particular PerceptOR (Perception for Off-Road robots) and LAGR (Learning Applied to Ground Robots). His roles included system engineering for the navigation system, research and development of constrained motion planning components, and culminated in leading the development of the navigation system for LAGR robots. Mihail also collaborated directly with the principal investigator in motion planning research as part of a Mars Technology Program task. Mihail's areas of research interest primarily include motion planning, modeling and system identification, and state estimation. 